\title{
Timing for an Integrated Accelerograph EDAS-AS with GPS and NTP
}

$$
\text { Chaoyong } \mathrm{PENG}^{1, \mathrm{a}} \text {, Bing XUE }{ }^{2, \mathrm{~b}} \text {, Jiansi } \mathrm{YANG}^{1, \mathrm{c}}
$$

\author{
${ }^{1}$ Institute of Geophysics, China Earthquake Administration, Beijing, 100081, China \\ ${ }^{2}$ Institute of Earthquake Science, China Earthquake Administration, Beijing, 100036, China

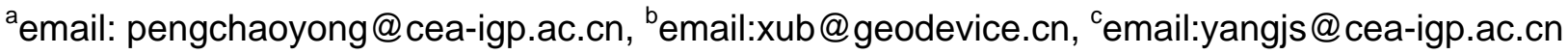

Keywords: Integrated Accelerograph; Time Error; Crystal Oscillator Adjustment; GPS; NTP

\begin{abstract}
In order to solve problems of “jump second” when using only GPS for timing service and inconvenience of installing GPS antennas in some places, we propose a new algorithm combining GPS and NTP to adjust the local clock of an integrated accelerograph. The process flow of the method was described in detail, and the timing accuracy was tested. When the local clock is synchronized against the GPS time output, the time accuracy can reach to $10 \mu \mathrm{s}$, while if it is only synchronized against NTP servers, we can obtain a time accuracy of 2 ms. Moreover, during the long run test of the system, we have not found the problem of "jump second". Therefore, this method can effectively solve the problems when only using GPS for time service and the time accuracy can also meet the requirements of strong-motion observation on time precision.
\end{abstract}

\section{Introduction}

Timing for earthquake events has always been one of the key technologies in seismological observation. During the period of analog seismological observation, when an earthquake happens, short wave is used to set time of an event, while in the digital seismological observation era, Global Positioning System (GPS) is adopted for identifying the time of an event [1]. Many seismic digitizers produced both at home and abroad, such as EDAS-24GN [2-4] (China), REFTEK 130-01 [5] (America), Q0330 [6] (America) and Guralp CMG-DM24 [7] (England), have built-in GPS receivers which can provide high precision timing signals for the digitizers to ensure the absolute accuracy of microsecond time.

However, there are inevitable two problems existed in such seismic data acquisition systems using only GPS for time service. One is occasional second fluctuation, namely "jump second", which may be caused by pps (pulse per second) signal fluctuation derived from a GPS receiver's output or "leap second". The other is that it is inconvenient to install GPS antennas in some places such as in a dam. For these places, a very long GPS cable is needed. However, for these observations, requirements of data time precision is not very high and millisecond level is enough for time service.

Therefore, in order to solve these two problems, we propose a new time service method with GPS and NTP (Network Time Protocol) [8] for an integrated accelerograph EDAS-AS. NTP is a type of protocol with rfc number 1305 and can be used to synchronize timekeeping among a set of distributed time servers and clients.

\section{Method}

The main flow of the new time service method with GPS and NTP is shown in Figure 1, and can be divided into four cases classified according to GPS/NTP state synchronous flag (Table 1). 
Table 1. Four cases distinguished with GPS/NTP state synchronous flag

\begin{tabular}{cll}
\hline Case & \multicolumn{1}{c}{ GPS state } & \multicolumn{1}{c}{ NTP state } \\
\hline 1 & Synchronized & Synchronized \\
2 & Synchronized & Unsynchronized or no network \\
3 & Unsynchronized or no GPS receiver & Synchronized \\
4 & Unsynchronized or no GPS receiver & Unsynchronized or no network \\
\hline
\end{tabular}

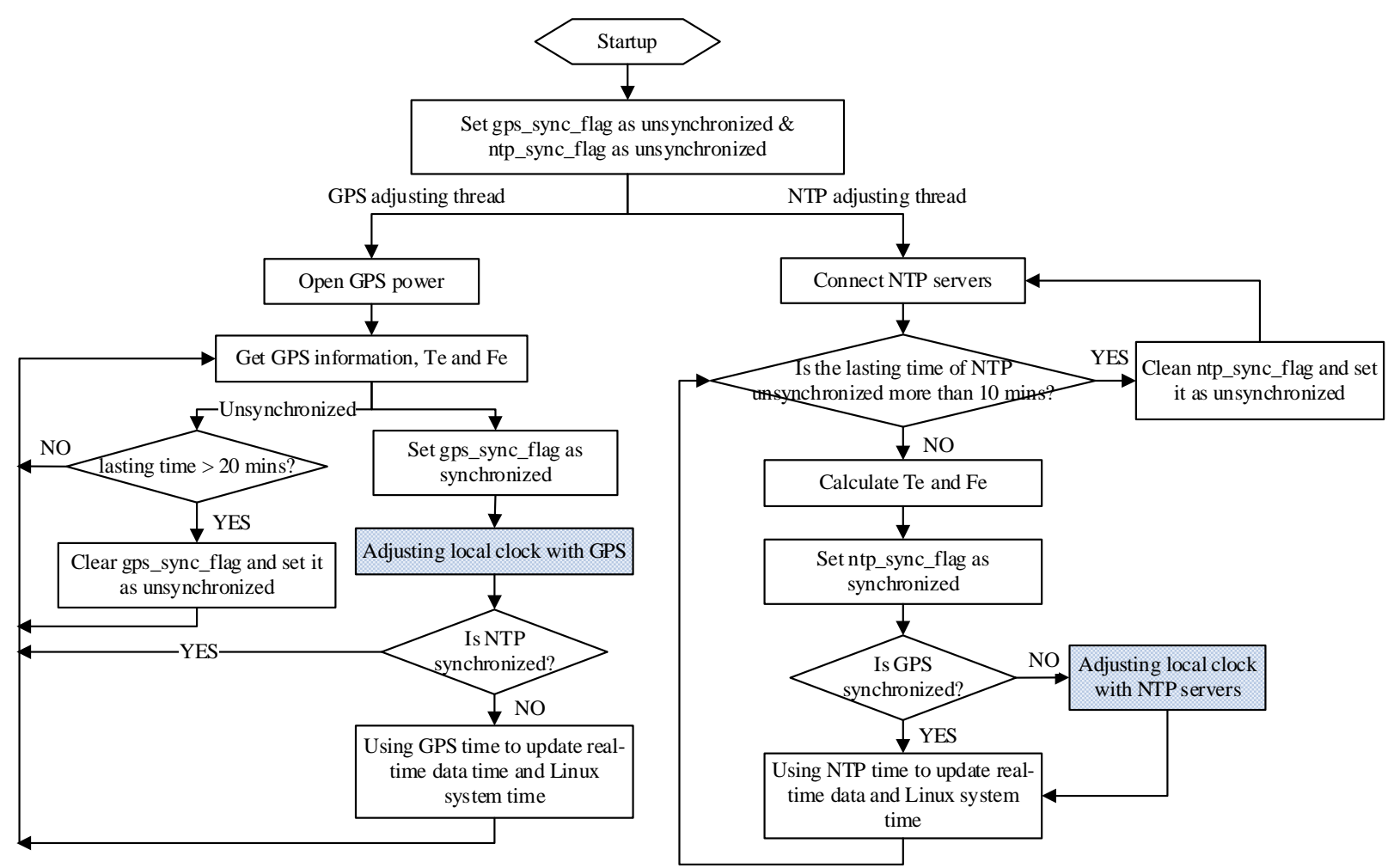

Fig.1. Main flow of time service with GPS and NTP

For the first case, the absolute time obtained from NTP servers is used to set the real-time data time and Linux system time, while time error Te with the order of $\mu$ seconds and frequency error $\mathrm{Fe}$ of the internal oscillator are adjusted according to the buffered oscillator value recorded at each pps.

The second case is the same as the traditional method using only GPS for time service, where the absolute time obtained from GPS is used to set the real-time data time and Linux system time, and Te and Fe of the internal oscillator are adjusted according to the buffered oscillator value read at each pps.

For the third case, only time information acquired from NTP servers is used because GPS cannot be synchronized or there is no GPS receiver connecting with the data acquisition system. We use this time information to set the real-time data time and Linux system time, and the means to adjust $\mathrm{Te}$ and $\mathrm{Fe}$ is consistent with the first case.

The last one has no GPS and NTP time information. For this case, the latest stored DA voltage is used to control stability of the oscillator with an interval of 20 minutes.

\section{Time Service Algorithm with GPS}

To provide accurate time service for the integrated accelerograph, we designed a hardware structure to process GPS time information [9], as shown in Figure 2. When the GPS receiver is synchronized with the GPS satellite systems, an IRIG-B code and a pps signal will be outputted through it. After analyzing the IRIG-B code, we can obtain time information including year, month, day, hour, minute, second, etc. These information are combined to form the absolute time and provide time service for the whole system. Meanwhile, the pps signal is exploited by the FPGA to latch the count value of crystal oscillator with $8.192 \mathrm{MHz}$, and the latched value is simultaneously 
sent to the ARM CPU through a synchronous serial controller (SSC). The value will be combined with the latest stored ones for statistical analysis. Then, we can calculate Te and Fe from the analytic results. These two errors will be converted to a DA value used to adjust frequencies of the voltage controlled crystal oscillator.

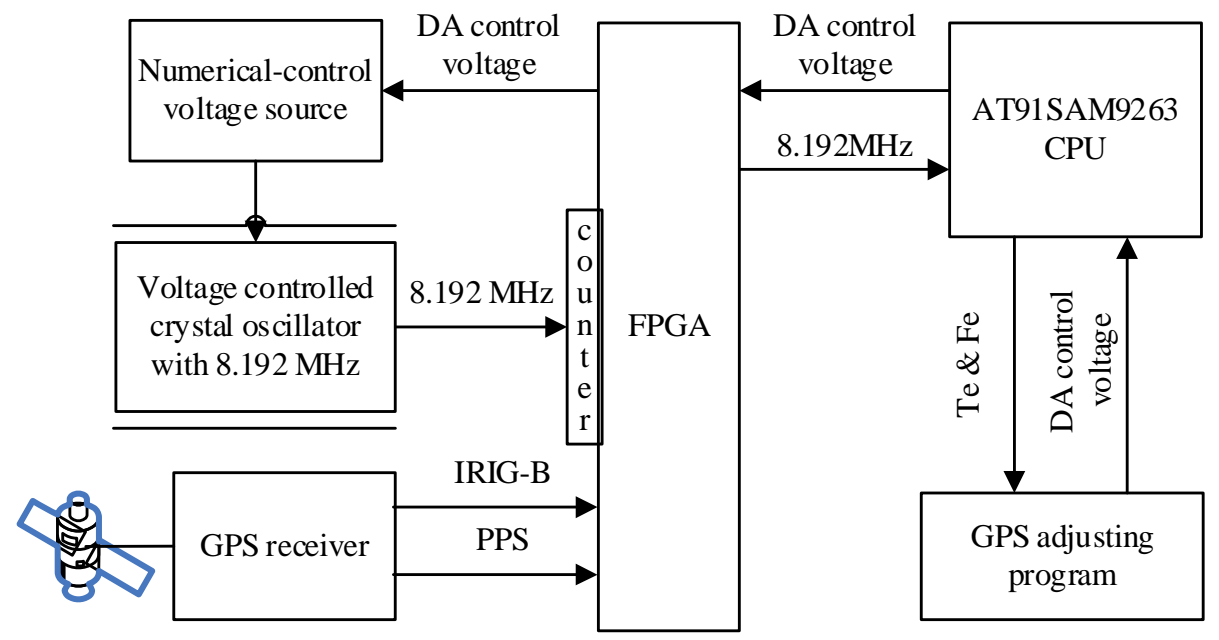

Fig.2. Hardware structure for processing GPS information

Determination of Te and Fe. When latching count values of the crystal oscillator with 8.192 $\mathrm{MHz}$, abnormal values caused by pps signal jitter need to be excluded [10]. In the proposed algorithm, if difference between the current value and the cache values is greater than a predefined threshold, the current value will be removed. Because we have used two buffers to cache the count values (one is with 10 values, and the other has 50 values), threshold values for these two buffers are different from each other. For the buffer with 10 values, the threshold of consecutive 6 values is $1000 \mathrm{~Hz}$, while for the one with 50 values, $2500 \mathrm{~Hz}$ is set as the threshold of consecutive 15 values.

How to calculate $T_{e}$ is relatively easy and only the current latched value is used to obtain this value as follows,

$$
\begin{aligned}
& T_{e}=C / 8 \\
& T_{e}=C / 8-1024000
\end{aligned}
$$

where $T_{e}$ is time error which has been transferred to the order of $\mu \mathrm{s}, C$ is the current value of 8.192 $\mathrm{MHz}$ latched by pps signal. Equation (1a) is adopted when the crystal oscillator is faster, while equation (1b) is used when the crystal oscillator is slower.

For calculating $\mathrm{F}_{\mathrm{e}}$, moving average filter method is adopted in order to eliminate the effect of pps signal fluctuation. The formula is

$$
F_{e}=\frac{\frac{1}{n} \sum_{i=2}^{n+1} T_{i}-\frac{1}{n} \sum_{i=1}^{n} T_{i}}{\lambda}=\frac{T_{n+1}-T_{1}}{n \lambda}
$$

where $F_{e}$ is frequency error, $T_{n+1}$ is the current latched value, $T_{i}(i: 1$ to $\mathrm{n}$ ) is the cached values, $\lambda$ is the sampling time ( $1 \mathrm{~s}), n$ is the length of the sliding window (10 or 50). The frequency error with unit $\mathrm{Hz}$ will be converted to value with unit $\mathrm{ppb}\left(8.192 \mathrm{~Hz}=1 \mathrm{PPM}=10^{3} \mathrm{ppb}\right)$ by

$$
F_{e}^{\prime}=F_{e} \times 1000 / 8.192
$$

Process for adjusting the crystal oscillator with GPS. The process flow for synchronizing local clock of the integrated accelerograph with GPS clock is shown in Figure 3. To improve the efficiency, the whole process has been divided into three stages.

The first stage is at the time after the system startup. At this stage, the time difference between system time and GPS time is random. In order to accelerate the process of synchronization, we instantly clear the frequency counter to set the error in the range of $10 \mathrm{~ms}$, and the absolute time and date are updated by the GPS input.

Then, we will enter the second stage. At this stage, the buffer with 10 values is used to adjust 
frequency of the crystal oscillator. Different control ranges are defined (Table 2) to adjust $\mathrm{T}_{\mathrm{e}}$ and $\mathrm{F}_{\mathrm{e}}$ for improving efficiency of the synchronization process. When $T_{e}$ is lower than $30 \mu \mathrm{s}$ and $\mathrm{F}_{\mathrm{e}}$ is smaller than $100 \mathrm{ppb}$, the process will jump into the third stage.

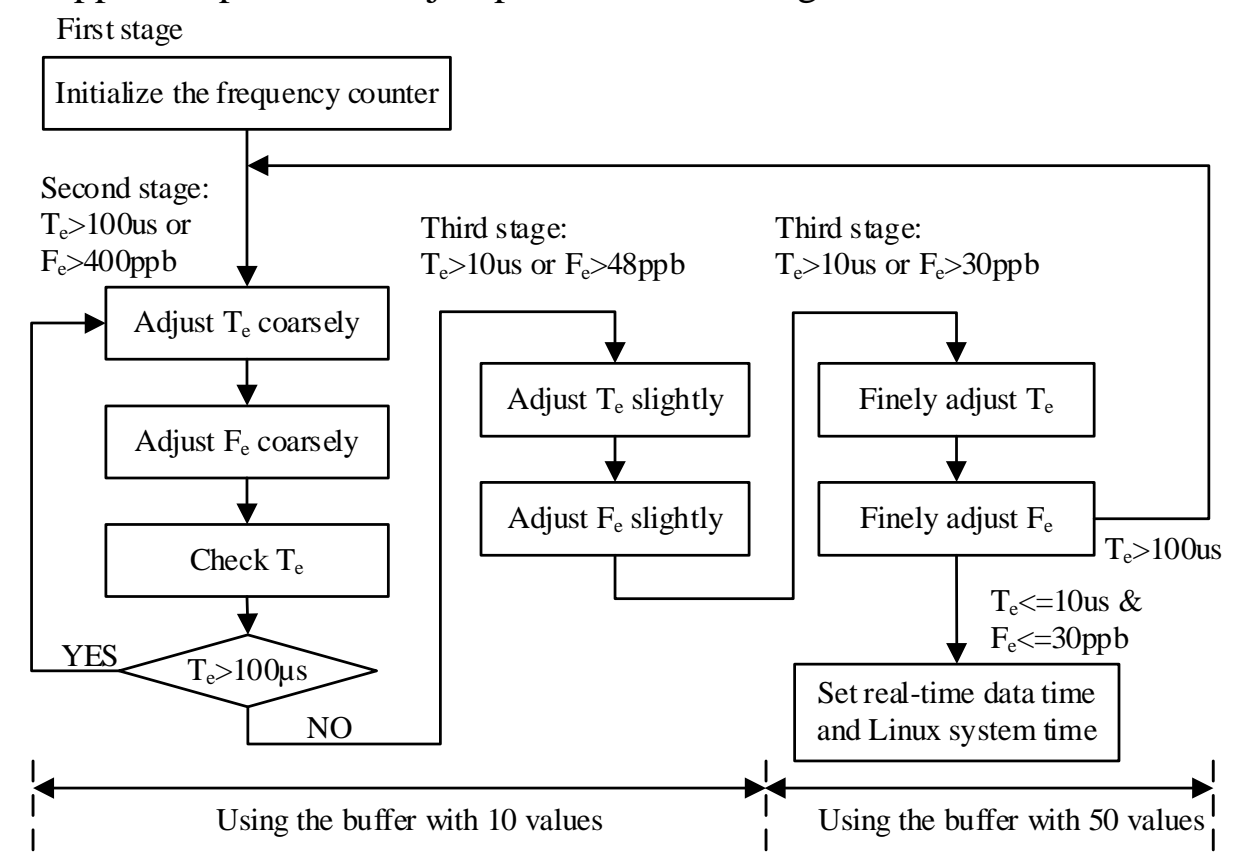

Fig.3. Flow for adjusting the crystal oscillator only with GPS

Table 2. Different control ranges for $\mathrm{T}_{\mathrm{e}}$ and $\mathrm{F}_{\mathrm{e}}$ used by GPS adjustment

\begin{tabular}{ll}
\hline \multicolumn{1}{c}{ Control ranges for $\mathrm{T}_{\mathrm{e}}$} & \multicolumn{1}{c}{ Control ranges for $\mathrm{F}_{\mathrm{e}}$} \\
\hline $\mathrm{T}_{\mathrm{e}} \in(500 \mu \mathrm{s}, 10 \mathrm{~ms}]$ & $\mathrm{F}_{\mathrm{e}}>200 \mathrm{ppb}$ \\
$\mathrm{T}_{\mathrm{e}} \in[-10 \mathrm{~ms},-500 \mu \mathrm{s})$ & $\mathrm{F}_{\mathrm{e}}<-200 \mathrm{ppb}$ \\
$\mathrm{T}_{\mathrm{e}} \in(120 \mu \mathrm{s}, 500 \mu \mathrm{s}]$ & $\mathrm{F}_{\mathrm{e}} \in(100 \mathrm{ppb}, 200 \mathrm{ppb}]$ \\
$\mathrm{T}_{\mathrm{e}} \in[-500 \mu \mathrm{s},-120 \mu \mathrm{s})$ & $\mathrm{F}_{\mathrm{e}} \in[-200 \mathrm{ppb},-100 \mathrm{ppb})$ \\
$\mathrm{T}_{\mathrm{e}} \in(30 \mu \mathrm{s}, 120 \mu \mathrm{s}]$ & $\mathrm{F}_{\mathrm{e}} \in[-100 \mathrm{ppb}, 100 \mathrm{ppb}]$ \\
$\mathrm{T}_{\mathrm{e}} \in[-120 \mu \mathrm{s},-30 \mu \mathrm{s})$ & \\
$\mathrm{T}_{\mathrm{e}} \in[-30 \mu \mathrm{s}, 30 \mu \mathrm{s}]$ & \\
\hline
\end{tabular}

For the third stage, we use the buffer with 10 values to adjust frequency of the oscillator until $\mathrm{T}_{\mathrm{e}}$ is lower than $10 \mu \mathrm{s}$ and $\mathrm{F}_{\mathrm{e}}$ is smaller than $48 \mathrm{ppb}$. Then, the buffer with 50 values is adopted for finely tuning the oscillator's frequency.

\section{Time Service Algorithm with NTP}

The overall organization of the NTP architecture shown in Figure 4 is divided into four parts: NTP time server, receiver process module (RPM), update process module (UPM) and local clock process module (LCPM). 


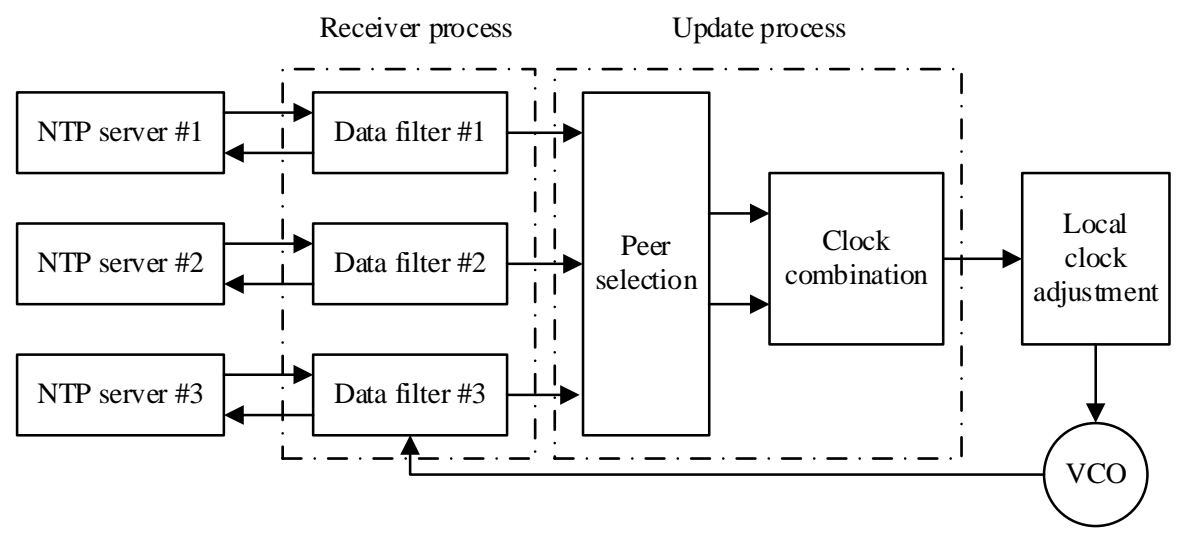

Fig.4. Overall organization of the NTP architecture

The time server used as a synchronization source is absolutely necessary for adjusting the local clock. When a time server receives an NTP message sent by an integrated accelerograph, it will interchange addresses and ports, overwrite certain fields in the message, recalculate the checksum and return the message immediately. Usually, more than one time sever are utilized to synchronize system time of the accelerograph.

When an NTP message is returned to the accelerograph, the RPM will calculate $T_{e}$ between the peer clock and the local clock and incorporate the result into the data base along with other information useful for error determination and peer selection. A filtering algorithm is used to improve the accuracy by discarding inferior data.

The UPM is initiated upon receipt of a message and at other times. It processes $T_{e}$ from each peer and selects the best one using peer selection algorithm and clock combining algorithm mentioned in [8]. This may involve many observations of a few peers or a few observations of many peers.

The LCPM operates upon $\mathrm{T}_{\mathrm{e}}$ produced by the UPM and adjusts the phase and frequency of the crystal oscillator using the mechanisms shown in Figure 5. This may result in either a step-change or a gradual phase adjustment of the crystal oscillator to reduce $T_{e}$ to zero.

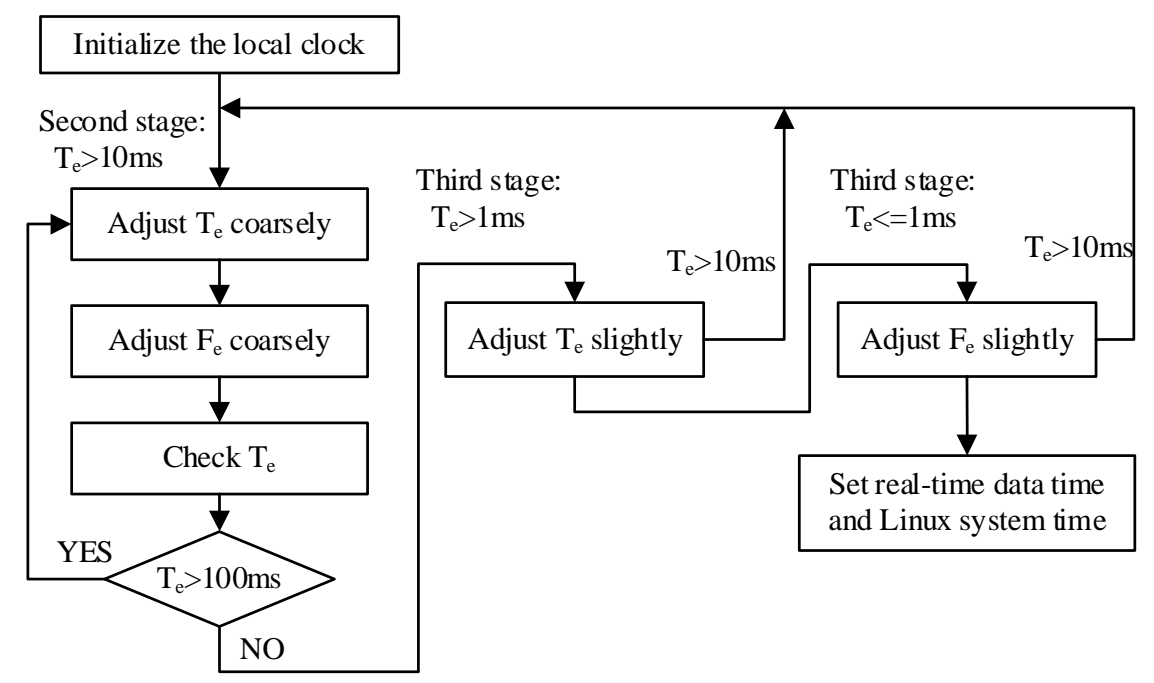

Fig.5. Flow for adjusting the crystal oscillator with NTP

Here, the definition of $\mathrm{F}_{\mathrm{e}}$ is different from the one using GPS for clocking, because there is no count value of $8.192 \mathrm{MHz}$ latched by pps signal. We set $\mathrm{F}_{\mathrm{e}}$ as the difference between the current $\mathrm{T}_{\mathrm{e}}$ and the last one and the unit is $\mu$ s. The flow shown in Figure 5 is similar to that of GPS adjustment. However, the control ranges (Table 3) are much wider than those used for GPS adjustment because of the relatively low accuracy of NTP time. 
Table 3. Different control ranges for $\mathrm{T}_{\mathrm{e}}$ and $\mathrm{F}_{\mathrm{e}}$ used by NTP adjustment

\begin{tabular}{ll}
\hline \multicolumn{1}{c}{ Control ranges for $\mathrm{T}_{\mathrm{e}}$} & \multicolumn{1}{c}{ Control ranges for $\mathrm{F}_{\mathrm{e}}$} \\
\hline $\mathrm{T}_{\mathrm{e}}>20 \mathrm{~ms}$ & $\mathrm{~F}_{\mathrm{e}}>5 \mathrm{~ms}$ \\
$\mathrm{~T}_{\mathrm{e}}<-20 \mathrm{~ms}$ & $\mathrm{~F}_{\mathrm{e}}<-5 \mathrm{~ms}$ \\
$\mathrm{~T}_{\mathrm{e}} \in[-20 \mathrm{~ms},-10 \mathrm{~ms})$ & $\mathrm{F}_{\mathrm{e}} \in(1 \mathrm{~ms}, 5 \mathrm{~ms}]$ \\
$\mathrm{T}_{\mathrm{e}} \in[-20 \mathrm{~ms},-10 \mathrm{~ms})$ & $\mathrm{F}_{\mathrm{e}} \in[-5 \mathrm{~ms},-1 \mathrm{~ms})$ \\
$\mathrm{T}_{\mathrm{e}} \in(6 \mathrm{~ms}, 10 \mathrm{~ms}]$ & $\mathrm{F}_{\mathrm{e}} \in[-1 \mathrm{~ms}, 1 \mathrm{~ms}]$ \\
$\mathrm{T}_{\mathrm{e}} \in[-10 \mathrm{~ms},-6 \mathrm{~ms})$ & \\
$\mathrm{T}_{\mathrm{e}} \in[-6 \mathrm{~ms}, 6 \mathrm{~ms}]$ & \\
\hline
\end{tabular}

\section{Test of Timing Accuracy}

After completion of the algorithm development, we separately tested the timing accuracy with GPS and that with NTP.

Timing Accuracy Test with GPS. The variation curves of $T_{e}$ and $F_{e}$ after the integrated accelerograph startup are shown in Figure 6. We can find that the initial $T_{e}$ and $F_{e}$ are larger and have more intense changes. The reason is that the synchronization process is at the second stage with large oscillator frequency adjustment. After about 10 minutes, the synchronization process enters the third stage, changes of $T_{e}$ and $F_{e}$ are gradually stable and the adjustment of the crystal oscillator frequency is very small.

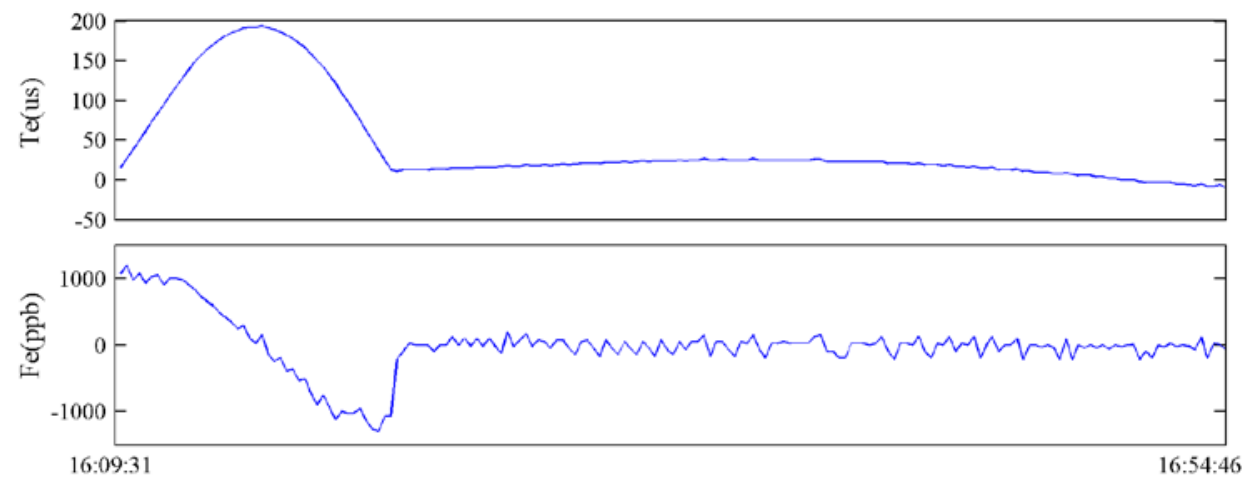

Fig.6. Variation curves of $\mathrm{T}_{\mathrm{e}}$ and $\mathrm{F}_{\mathrm{e}}$ after the instrument startup

Then, we unplugged the GPS cable. After 16 hours, we reconnected the cable with the instrument. The results are shown in Figure 7. For this case, the local clock has an offset of about 1 ms after 16 hours with no GPS cable. When readjusting frequency of the crystal oscillator, we did not need to clear the frequency counter because the initial $\mathrm{T}_{\mathrm{e}}$ did not exceed $10 \mathrm{~ms}$. However, due to the relatively large $T_{e}$ and $F_{e}$, the synchronization process expended about 25 minutes at the second stage before jumping into the third stage.

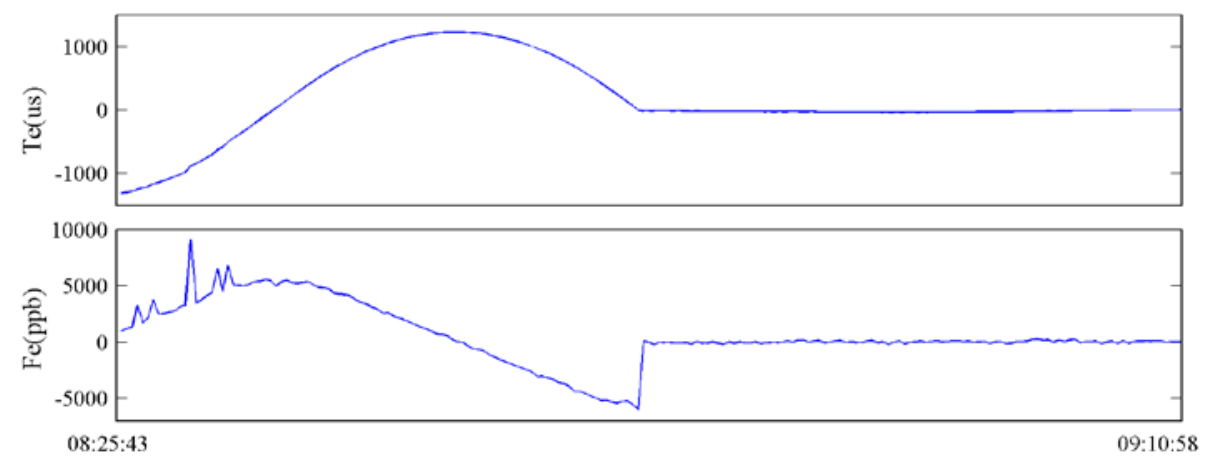

Fig.7. Variation curves of $\mathrm{T}_{\mathrm{e}}$ and $\mathrm{F}_{\mathrm{e}}$ after 16 hours with no GPS cable and then reconnecting the GPS cable with the instrument 


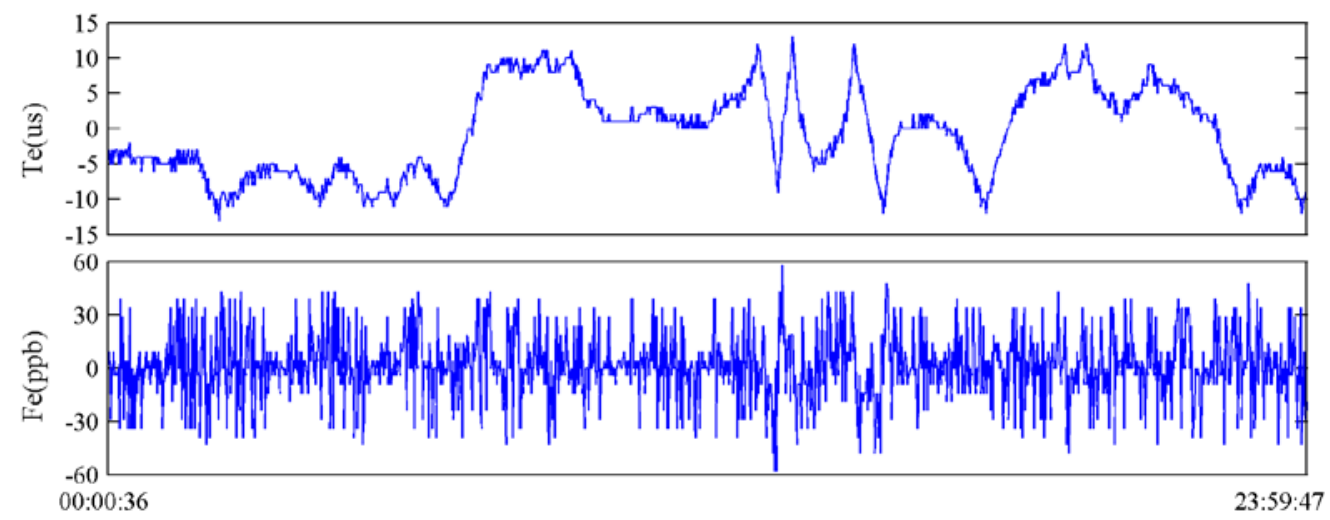

Fig.8. Variation curves of $\mathrm{T}_{\mathrm{e}}$ and $\mathrm{F}_{\mathrm{e}}$ within 24 hours after the local clock of the integrated accelerograph was synchronized against the GPS output

Figure 8 shows variation curves of $\mathrm{T}_{\mathrm{e}}$ and $\mathrm{F}_{\mathrm{e}}$ within 24 hours after the local clock of the integrated accelerograph was synchronized against the GPS output. As shown in Figure 8, the variation range of $T_{e}$ is between $\pm 15 \mu$ s and most of the time the error lies in a $\pm 10 \mu$ s range. For $F_{e}$, the variation range is between $\pm 60 \mathrm{ppb}$ with most of the time lying in the range of $\pm 30 \mathrm{ppb}$. The results prove the robustness of timing accuracy with GPS.

Timing Accuracy Test with NTP. The configuration for testing timing accuracy with NTP is no GPS cable, three NTP servers within which one is located in LAN. After the instrument startup, we need relatively long time to adjust the local clock because of no clearance of the frequency counter and very large $T_{e}$ and $F_{e}$. Figure 9 shows the variation curves of $T_{e}$ and $F_{e}$ error within 31 hours after the local clock of the integrated accelerograph was synchronized against the NTP servers. As shown in Fig. 9, the variation ranges of $T_{e}$ and $F_{e}$ are between $\pm 2 \mathrm{~ms}$ and $\pm 30 \mu$ s, respectively. The accuracy can meet the requirements of strong-motion observation on time precision.

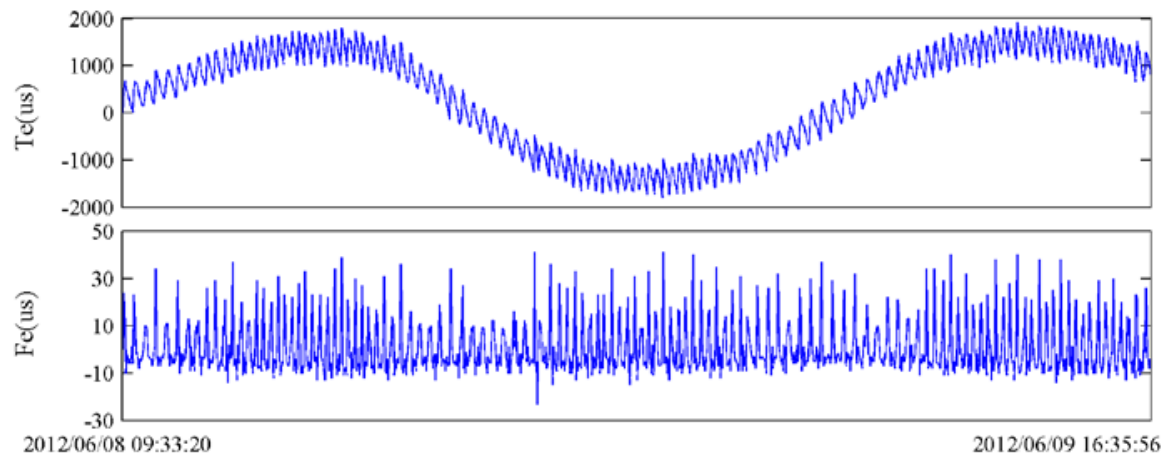

Fig.9. Variation curves of $\mathrm{T}_{\mathrm{e}}$ and $\mathrm{F}_{\mathrm{e}}$ within 31 hours after the local clock of the integrated accelerograph was synchronized against the NTP servers

\section{Conclusion}

In order to solve problems of "jump second" and inconvenience of installing GPS antennas in some places, we propose a new time service method combining GPS and NTP to adjust the local clock of an integrated accelerograph. The process flow of the method was described in detail, and the timing accuracy was tested. When the local clock is synchronized against the GPS time output, the time accuracy can reach to $10 \mu \mathrm{s}$, while if it is only synchronized against NTP servers, we can obtain a time accuracy of $2 \mathrm{~ms}$. Moreover, during the long run test of the system, we have not found the problem of "jump second". Therefore, this method can effectively solve the problems when only using GPS for time service and the time accuracy can also meet the requirements of strong-motion observation on time precision. 


\section{Acknowledgement}

This work was financially supported by the National Natural Science Foundation of China (41404048) and Basic Research Project of Institute of Geophysics, China Earthquake Administration (DQJB14B05).

\section{References}

[1] Cuifang Wang, Xiaoyuan Yang, Cheng Song, Yuping Shao. Technology of GPS timing and correcting-time for earthquake data acquisition device [J]. Seismological and Geomagnetic Observation and Research, 2010 31(1) 57-61.

[2] EDAS-24GN. http://www.geodevice.cn/p.aspx?id=2\&t=3.

[3] Chaoyong Peng, Xiaoyi Zhu, Jiansi Yang, Bing Xue, Yang Chen. Development of an integrated onsite earthquake early warning system and test deployment in Zhaotong, China [J]. Computers \& Geosciences, 201356 170-177.

[4] Chaoyong Peng, Jiansi Yang, Bing Xue, Yang Chen, Jiang Li, Xiaoyi Zhu, Zhiqiang Xu, Yu Zheng. A low-latency data acquisition system for earthquake early warning [J]. Earthquake, 2015 35(1) 140-148.

[5] Reftek 130-01. http://www.reftek.com/pdf/130-01.pdf.

[6] Q0330. http://www.kmi.com/pdfs/q330Rev1.pdf.

[7] Guralp CMG-DM24. http://www.guralp.com/documents/DAS-D24-0004.pdf.

[8] David L. Mills. Network Time Protocol (Version 3) specification, implementation and analysis. Network Working Group Report RFC-1305, University of Delaware, March, 1992, 113 pp.

[9] Hongti Wang, Cantao Zhuang, Bing Xue, Jiang Li, Yang Chen, Xiaoyi Zhu, Wenyu Lou, Minghui Liu. A network communication and recording system for digital seismic observation [J]. Acta Seismologica Sinica, 2006 28(5) 540-545.

[10] Xianghong Lu, Rujun Chen, Zhanxiang He. The design of frequency calibration system for OCXO based FPGA [J]. Application of Electronic Technique, 20107 104-107. 